\title{
Borrelia burgdorferi Not Confirmed in Human-Biting Amblyomma americanum Ticks from the Southeastern United States
}

\author{
Ellen Y. Stromdahl, ${ }^{a}$ Robyn M. Nadolny, ${ }^{\text {a,b }}$ Jennifer A. Gibbons, ${ }^{\text {c,d }}$ Lisa D. Auckland, ${ }^{e}$ Mary A. Vince, ${ }^{\text {a }}$ Chad E. Elkins, ${ }^{a}$ \\ Michael P. Murphy, ${ }^{\text {a }}$ Graham J. Hickling, ${ }^{\text {f }}$ Mark W. Eshoo, ${ }^{\text {g }}$ Heather E. Carolan, ${ }^{\text {g }}$ Chris D. Crowder, ${ }^{\text {g }}$ Mark A. Pilgard, ${ }^{\text {h }}$ Sarah A. Hamer ${ }^{\text {e }}$ \\ U.S. Army Public Health Command, Aberdeen Proving Ground, Maryland, USA ; Department of Biological Sciences, Old Dominion University, Norfolk, Virginia, USA ; U.S. \\ Army Edgewood Chemical and Biological Center, Aberdeen Proving Ground, Maryland, USA'; Science and Technology Corp., Hampton, Virginia, USA ; Department of \\ Veterinary Integrative Biosciences, Texas A\&M University, College Station, Texas, USA ${ }^{\text {; }}$ Center for Wildlife Health, University of Tennessee Institute of Agriculture, Knoxville, \\ Tennessee, USA; Ibis Biosciences, Carlsbad, California, USA ; Division of Vector-Borne Diseases, Centers for Disease Control and Prevention, Fort Collins, Colorado, USA ${ }^{h}$
}

The predominant human-biting tick throughout the southeastern United States is Amblyomma americanum. Its ability to transmit pathogens causing Lyme disease-like illnesses is a subject of ongoing controversy. Results of previous testing by the Department of Defense Human Tick Test Kit Program and other laboratories indicated that it is highly unlikely that $A$. americanum transmits any pathogen that causes Lyme disease. In contrast, a recent publication by Clark and colleagues (K. L. Clark, B. Leydet, and S. Hartman, Int. J. Med. Sci. 10:915-931, 2013) reported detection of Lyme group Borrelia in A. americanum using a nested-flagellin-gene PCR. We evaluated this assay by using it and other assays to test 1,097 A. americanum ticks collected from humans. Using the Clark assay, in most samples we observed nonspecific amplification and nonrepeatability of results on subsequent testing of samples. Lack of reaction specificity and repeatability is consistent with mispriming, likely due to high primer concentrations and low annealing temperatures in this protocol. In six suspect-positive samples, Borrelia lonestari was identified by sequencing of an independent gene region; this is not a Lyme group spirochete and is not considered zoonotic. B. burgdorferi was weakly amplified from one pool using some assays, but not others, and attempts to sequence the amplicon of this pool failed, as did attempts to amplify and sequence B. burgdorferi from the five individual samples comprising this pool. Therefore, $B$. burgdorferi was not confirmed in any sample. Our results do not support the hypothesis that $A$. americanum ticks are a vector for Lyme group Borrelia infections.

T he vectors and etiologic agents of Lyme-like diseases in the southeastern United States are a subject of ongoing controversy $(1,2)$. In the United States, most Lyme disease is caused by infection with Borrelia burgdorferi sensu stricto, a bacterium that is phylogenetically within the B. burgdorferi sensu lato "Lyme group" of spirochetes vectored by hard ticks. The Lyme group also includes genospecies implicated as the etiologic agents of Lyme disease in other geographic regions, including B. garinii, B. afzelii, $B$. spielmanii, and B. valaisiana in Europe. Additional Lyme group genospecies continue to be described. In the United States, these include B. americana, B. andersonii, and B. carolinensis, all of unknown pathogenicity, and $B$. bissettii, which has been implicated in cases of human illness (1). In contrast to the Lyme group, the relapsing fever (RF) group spirochetes, many of which are vectored by soft ticks, are a separate phylogenetic cluster that includes agents associated with RF disease in humans. In the United States, genospecies within the RF group include $B$. hermsii, $B$. turicatae, and B. parkeri, vectored by soft ticks, and B. miyamotoi, B. davisii, and $B$. lonestari, vectored by hard ticks. $B$. hermsii is the main cause of tick-borne relapsing fever in the United States; B. turicatae and $B$. parkeri have also been associated with human disease (3). B. miyamotoi was recently implicated as a cause of human disease in the United States (4), and the pathogenicity of B. davisii is unknown. B. lonestari was provisionally implicated in a single case of early Lyme disease-like illness (erythema migrans), but subsequent investigation has not established it as a human pathogen (5-7).

The predominant human-biting tick throughout the Southeast is Amblyomma americanum, accounting for over $90 \%$ of tick bites in southeastern states $(8,9)$. Vector competency studies have con- cluded repeatedly that $B$. burgdorferi is unlikely to be transmitted by $A$. americanum (10-16), and a potent borreliacidal agent has been identified in A. americanum saliva $(17,18)$. From 1997 to 2010, the Department of Defense (DOD) Human Tick Test Kit Program (HTTKP), a tick identification and PCR testing service provided by the U.S. Army Public Health Command (USAPHC) for DOD personnel and dependents, tested 22,565 A. americanum ticks $(21,245$ adults and nymphs removed from humans, and 1,320 field-collected larvae) for borrelial agents of Lyme-like diseases using a series of nested- and real-time PCR assays. These ticks include representation across the majority of the geographic distribution of the species $(8,9)$.

HTTKP methods have evolved across the years in response to changing diagnostic capabilities and new public health threats (2, 19, 20). For example, in 2001, when B. lonestari, an RF group Borrelia species found in A. americanum, was tentatively linked to

Received 4 December 2014 Returned for modification 3 January 2015 Accepted 7 March 2015

Accepted manuscript posted online 18 March 2015

Citation Stromdahl EY, Nadolny RM, Gibbons JA, Auckland LD, Vince MA, Elkins CE, Murphy MP, Hickling GJ, Eshoo MW, Carolan HE, Crowder CD, Pilgard MA, Hamer SA. 2015. Borrelia burgdorferi not confirmed in human-biting Amblyomma americanum ticks from the southeastern United States. J Clin Microbiol 53:1697-1704. doi:10.1128/JCM.03454-14.

Editor: B. W. Fenwick

Address correspondence to Ellen Y. Stromdahl, Ellen.y.stromdahl.civ@mail.mil. Copyright $\odot$ 2015, American Society for Microbiology. All Rights Reserved. doi:10.1128/JCM.03454-14 
a case of southern tick-associated rash illness (STARI) (5), HTTKP testing was modified to include testing for B. lonestari (21-24). However, epidemiological studies of STARI patients did not support the hypothesized link between STARI and B. lonestari $(6,7,25)$, so targeting of this Borrelia species in the HTTKP ended in 2009. As a summary of our program from 1997 to 2010, a total of 4,019 A. americanum adults, nymphs, and larvae were tested with nested primers expected to amplify B. burgdorferi, and 7,421 were tested with primers that would have amplified all Borrelia spp. (23). Although we initially reported that seven samples (0.3\%), all from 1997, produced amplicons (19), we believe contamination was involved because all occurred within a short time period in the first year of our study when we initially implemented PCR techniques, and zero positive results were obtained across large numbers of samples in all subsequent years. In contrast, over the same period, a total of 5,458 Ixodes scapularis and 12 Ixodes pacificus adults and nymphs were tested by the HTTKP using equivalent assays: $24.7 \%$ of I. scapularis and $8.3 \%$ of I. pacificus ticks tested positive for Lyme group Borrelia DNA. To summarize, we found no measureable prevalence of B. burgdorferi in an epidemiologically relevant collection of $A$. americanum ticks from across a broad geographic range over a 13-year period.

In 2013, however, debate about the role of A. americanum in Lyme disease-like illness intensified, following a publication that reported detection of $B$. burgdorferi DNA in two $A$. americanum ticks removed from humans, and B. burgdorferi and B. andersonii DNA in humans bitten by A. americanum (26). Given our extensive background of PCR testing of $A$. americanum, and in view of the novelty and potential clinical significance of the suggestion by Clark et al. (26) that this tick species is contributing to unrecognized cases of human borreliosis in the southeastern United States, we used the primers and thermocycling protocol of Clark et al. (26) in an attempt to detect Lyme group spirochetes in a large sample $(>1,000)$ of $A$. americanum ticks removed from humans throughout the eastern United States in 2013.

\section{MATERIALS AND METHODS}

Tick identification and DNA extraction. A. americanum ticks were removed from military personnel and their families in the mid-Atlantic and southeastern United States and submitted to the HTTKP (Table 1). Submitted ticks were identified to species morphologically. Genomic DNA was then extracted either from individual ticks or from small pools of conspecific ticks removed from the same human on the same date, using a Zymo Genomic DNA II kit (Zymo Research Corporation, Orange, CA) (27). Aliquots of DNA from five A. americanum samples were pooled for a screening PCR; individual samples in these pools were archived for later PCR and/or sequencing confirmation if the pool tested suspect positive. Samples removed from the same person could contain more than one tick, so some pools contained DNA from more than five ticks.

PCR and sequence analysis. At our USAPHC laboratory, nested PCR for the flaB gene using primers from Clark et al. (26) was performed in $25-\mu l$ reaction volumes prepared with Ready-To-Go PCR beads (Amersham Pharmacia Biotech, Piscataway, NJ), containing $10 \mathrm{mM}$ Tris- $\mathrm{HCl}$ (pH 9.0), $1.5 \mathrm{mM} \mathrm{MgCl}_{2}, 200 \mu \mathrm{M}$ each deoxynucleotide triphosphate (dNTP), and 1.5 U Taq DNA polymerase. External reactions using outer reaction primers $280 \mathrm{~F}$ and $754 \mathrm{R}$ contained $2.5 \mu \mathrm{l}$ tick DNA $(0.5 \mu \mathrm{l}$ from individual ticks in pools of 5) and amplified a 475-bp product; internal reactions using inner reaction primers $301 \mathrm{~F}$ and $737 \mathrm{R}$ contained $1.0 \mu \mathrm{l}$ external PCR and amplified a 437-bp product. Of the two internal reaction primer sets published by Clark et al. (26), we used the set that provided the clearest gel results when tested with positive controls. This was the primer combination-280F, 754R and 301F, 737R_- used to amplify
TABLE 1 Origins by state of all ticks tested in this study

\begin{tabular}{ll}
\hline State & No. of ticks tested \\
\hline VA & 661 \\
MD & 151 \\
NJ & 53 \\
NC & 48 \\
KY/TN ${ }^{a}$ & 40 \\
KY & 33 \\
KS & 27 \\
TN & 24 \\
MO & 22 \\
DE & 14 \\
SC & 11 \\
AL & 4 \\
AR & 2 \\
FL & 2 \\
GA & 2 \\
FL/GA ${ }^{b}$ & 1 \\
RI & 1 \\
PA & 1 \\
& \\
Total & 1,097 \\
\hline${ }^{a}$ Ticks from Ft. Campbell, on the border of Kentucky and Tennessee, are listed as \\
"KY/TN." \\
${ }^{b}$ One tick was reported as acquired in either Florida or Georgia and is listed as \\
"FL/GA."
\end{tabular}

Lyme group Borrelia in a subsequent study of human samples by Clark et al. (28). Published thermal cycling parameters were followed $(26,28)$. Three positive controls were used, consisting of B. burgdorferi strain B31 (a gift from R. Wirtz, CDC, Atlanta, GA) and two B. burgdorferi-positive I. scapularis samples from the HTTKP. Each PCR included at least two negative controls, consisting of a water template sample that was introduced at the time of DNA extraction and a water template sample that was introduced at the time of PCR. Amplicons were visualized on $2 \%$ agarose gel cassettes using ethidium bromide (E-Gel; Invitrogen Corp., Carlsbad, CA). When a pool produced an amplicon at the 437-bp position, we then tested DNA from the individual tick samples that comprised the pool to ascertain individual-level infection, using the same method as described above.

At our Texas A\&M University (TAMU) laboratory, a blinded collection of flaB PCR-suspect-positive and -negative samples, along with positive and negative controls, was subjected to further analysis, following whole-genome amplification to increase the amount of template DNA available for testing (GenomiPhi; GE Healthcare, Pittsburgh, PA). This amplification process produces a concentrated DNA template, and therefore DNA was diluted 1:25 for subsequent analyses. First, the identification of tick species was confirmed through PCR and DNA sequencing of the 12 S rRNA gene (29). Next, a nested PCR for the 16S-23S rRNA intergenic spacer region (IGS) of Borrelia was performed, using primers and thermal cycling parameters described previously (30). This assay amplifies both RF and Lyme group borreliae, producing approximate 500-bp and 1,000-bp fragments, respectively. PCR amplicons from positive samples were purified (ExoSAP-IT; Affymetrix, Santa Clara, CA) and bidirectionally sequenced. Sequences were manually edited, and the identification of the Borrelia species was ascertained through comparison to sequences published in NCBI GenBank. Finally, real-time quantitative PCR (qPCR) was run on the samples using primers and probes for the 16S rRNA gene of Borrelia, including a B. burgdorferi-specific probe and an RF group probe (31).

At Ibis Biosciences, further testing of PCR-suspect-positive samples was undertaken, using isothermal Borrelia enrichment performed as previously published (32). Enrichment of a negative control was also performed. Following isothermal Borrelia DNA enrichment, a broad-range 
PCR followed by electrospray ionization mass spectrometry (IA/PCR/ ESI-MS) was performed, in which each sample was subjected to a series of eight diagnostic PCRs $(32,33)$. By the selection of Borrelia PCR targets that vary in sequence between Borrelia species and strains (genotypes), it is possible to use the IA/PCR/ESI-MS technology to identify the Borrelia species and distinguish its genotype even when present in mixtures of genotypes $(32,33)$. A volume of $10 \mu$ l of isothermal amplified nucleic acid extract was used in each PCR, and all PCRs were analyzed using an electrospray ionization mass spectrometry system (Abbott Molecular, Des Plaines, IL) as previously described $(32,33)$.

In silico analysis. Potential mispriming genomic loci were identified for each primer assay of Clark et al. (26) using NCBI Primer-BLAST (http: //www.ncbi.nlm.nih.gov/tools/primer-blast) default parameters against the nr database (a nonredundant NCBI database that includes all GenBank, RefSeq Nucleotides, EMBL, DDBJ, and PDB sequences, excluding HTGSO, 1, and 2, EST, GSS, STS, PAT, and WGS). The species of each potential mispriming site was noted for each assay.

The flagellin sequences of experimentally identified potential crossreacting Borrelia species were aligned using Clustalw (http://www .genome.jp/tools/clustalw/). The sequences used were from B. burgdorferi B31 (NC_001318.1), B. andersonii (D83762.1), B. americana SCW-30 h (HM802232.1), and B. lonestari MO2002-V1 (AY850063.1). The B. andersonii, B. americana, and B. lonestari genomes are not yet fully assembled, so the longest flagellin sequences deposited in NCBI Nucleotide were chosen for the alignment.

The melting temperatures $\left(T_{m} \mathrm{~s}\right)$ for each primer assay were determined using an online calculator (http://www.idtdna.com/analyzer /Applications/OligoAnalyzer/), adjusting default parameters to represent our PCR conditions $(0.8 \mu \mathrm{M}$ primers, $200 \mu \mathrm{M}$ dNTPs, and $1.5 \mathrm{mM}$ $\mathrm{MgCl}_{2}$ ) and identified mismatches.

Sensitivity test. PCRs were performed at USAPHC to compare the sensitivity of the flaB primers from Clark et al. (26) and the set of nested Borrelia genus fla primers from Barbour et al. (23) routinely used in the HTTKP. Serial dilutions of B. americana SCW-30E, B. andersonii SI-10, and B. bissettii FD-1 were tested with the two PCRs. These particular strains are from the southern United States and were chosen in order to more rigorously test the sensitivity of these primers and their ability to detect all members of these species, because these strains varied the most with the Barbour primers. To identify mismatches between the Barbour fla primers and B. americana, B. andersonii, and B. bissettii strains from the United States, an alignment of the flagellin sequences found in NCBI GenBank was made using the MegAlign application in the DNAStar Lasergene 12 software. The protocol for PCR using the Clark primers is described above, and the PCR protocol for the Barbour primers is described by Stromdahl et al. (3). Each test contained a positive control containing B. burgdorferi strain B31 and a negative control consisting of a water template sample that was introduced at the time of the PCR.

\section{RESULTS}

USAPHC testing. PCR using the nested flaB primers from Clark et al. (26) was performed on 171 pools, comprising 1,097 individual $A$. americanum ticks. The geographic origin of these ticks is listed in Table 1, and the results of testing are summarized in Table 2. The B31 positive controls and the two I. scapularis samples previously PCR positive for $B$. burgdorferi all produced major bands at $437 \mathrm{bp}$. In contrast, in most $A$. americanum samples, we observed only a "ladder" effect (Fig. 1) that typically is indicative of mispriming. For the few samples that produced a distinct major band of the expected length (e.g., Fig. 1A, lane 7, and B, lane 8), we classified the sample as suspect positive and investigated further. Nine of 171 pools (5.3\%)—P009, P084, P123, P171, P174, P189, P191, P194, and P211-were selected by this criterion.

Additional testing was conducted on the individual samples comprising the nine pools that produced major bands of the 437-bp size. Two of these pools (P009 and P171) yielded no suspect-positive individual samples. Accordingly, we repeated the flaB PCR on these and the remaining pools before testing the individual samples from any more pools. The pools P009 and P171, with no suspect-positive individual samples, and three additional pools, P189, P191, and P211, were negative in the repeat PCR of the pool, so the individual samples from P189, P191, and P211 were not initially tested using the flaB primers. The five individual samples from P189 were later tested (see "Follow-up testing at USAPHC and TAMU” below). Four pools (P084, P123, $\mathrm{P} 174$, and P194) contained individual samples that produced a 437-bp amplicon. Three pools, P084, P123, P194, contained one positive tick each, and one pool, P174, contained two positive ticks. Overall, 5 of 1,097 (0.46\%) individual A. americanum ticks (130786, 131114, 131429, 131433, and 131566) produced major bands of $437 \mathrm{bp}$. These five samples were submitted from Rhode Island, Virginia, Tennessee, and New Jersey.

TAMU testing. The five individual samples (130786, 131114, 131429, 131433, and 131566) and eight pools (P009, P123, P171, P174, P189, P191, P194, and P211) producing major bands of 437 bp in the flaB PCR were sent to the TAMU laboratory for further analysis. The pool (P084) containing the individual suspect-positive sample from Rhode Island was not sent. The $12 \mathrm{~S}$ rRNA sequence analysis revealed that all samples except one (130786 from P084), contained DNA from A. americanum. Sample 130786, was molecularly identified as I. scapularis and so had been misidentified by morphology at USAPHC as A. americanum. This tick sample, which had been removed from a human in Rhode Island, was retained as a positive control in subsequent analyses and was confirmed as positive for B. burgdorferi in all subsequent assays.

In the IGS PCR, six of eight pools (P123, P171, P174, P189, P194, and P211) produced amplicons, including five amplicons (P123, P171, P174, P194, and P211) that were approximately 500 bp (indicative of RF group spirochetes) and one very faint amplicon (P189) that was approximately $1,000 \mathrm{bp}$ (indicative of Lyme group spirochetes) (Table 2). Additionally, all four individual $A$. americanum ticks $(131114,131429,131433$, and 131566) produced amplicons approximately $500 \mathrm{bp}$ in size, and all four were associated with pools producing amplicons approximately $500 \mathrm{bp}$ in size. DNA sequencing of these 500-bp IGS amplicons in each case revealed the presence of $B$. lonestari (Table 2 ). The sequence reaction failed for the faint 1,000-bp IGS amplicon from pool P189.

In the qPCR, six (P123, P171, P174, P189, P194, and P211) of eight pools were considered suspect positive. Consistent with the IGS results reported above, five pools (P123, P171, P174, P194, and P211) were positive with the RF group probe $\left(C_{T}\right.$ values ranged from 32 to 35$)$, and one (P189) was weakly positive with the Lyme group Borrelia probe $\left(C_{T}\right.$ value of 39$)$ (Table 2$)$. Additionally, all four individual A. americanum ticks (131114, 131429. 131433 , and 131566) were positive with the RF group probe $\left(C_{T}\right.$ values ranged from 31 to 33 ), consistent with the IGS results that indicated the presence of $B$. lonestari DNA in these samples.

IA/PCR/ESI-MS analysis. Nine pools (P009, P084, P123, P171, P174, P189, P191, P194, and P211) and four individual samples $(131114,131429,131433$, and 131566) producing a major band of 437 bp in the flaB PCR assay and four pools (P184, P187, P20, and P211) and five individual samples (131431, 131559, 131560,131570 , and 131571) that were negative in that assay were 
TABLE 2 Analysis of A. americanum ${ }^{a}$ samples with bright bands at 437 bp in the B. burgdorferi flaB PCR of Clark et al. $(26)^{b}$

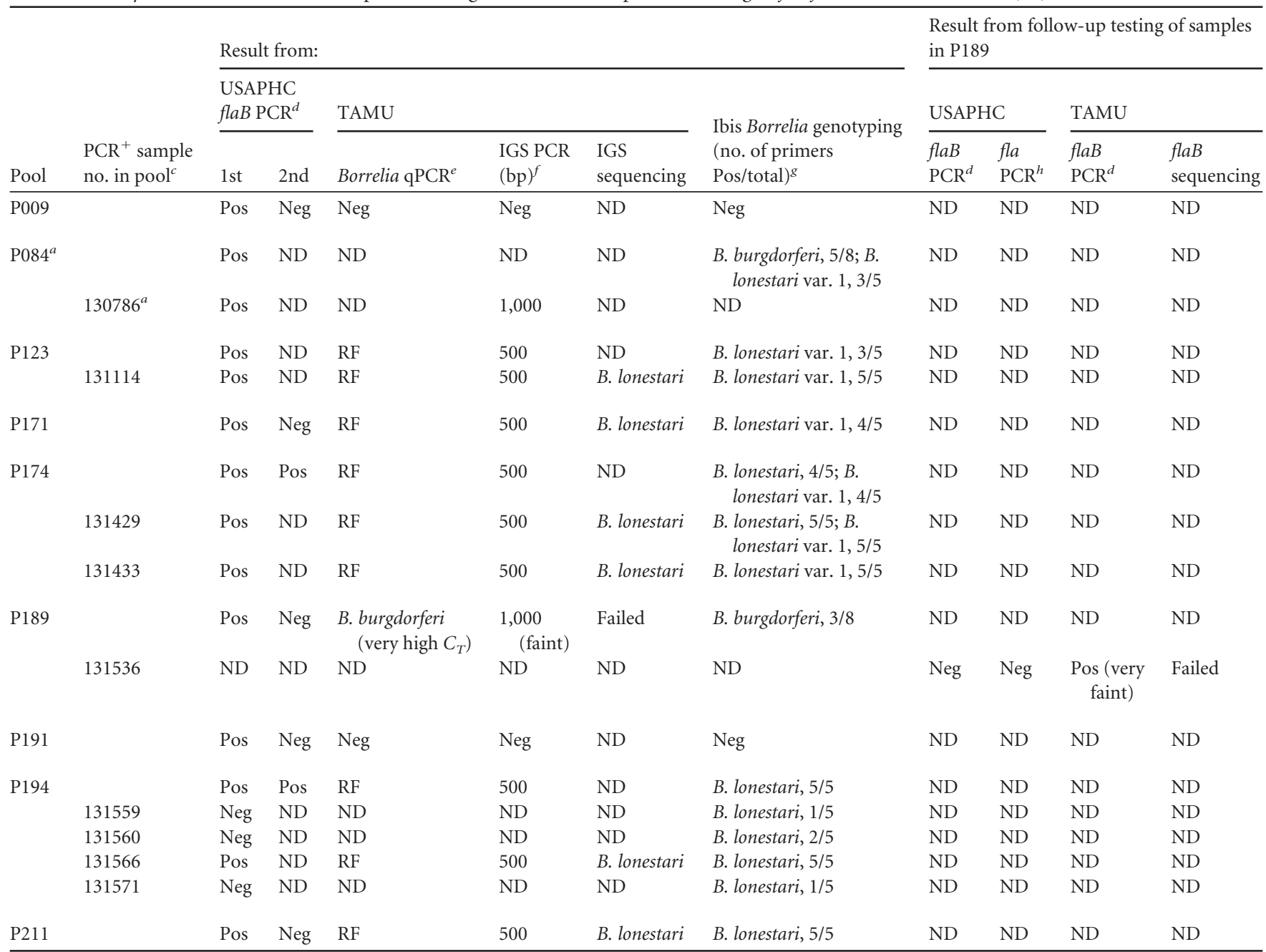

${ }^{a}$ Sample 130786 in P084 had been initially misidentified as A. americanum at USAPHC and was later molecularly identified as I. scapularis at TAMU.

${ }^{b}$ USAPHC, U.S. Army Public Health Command; TAMU, Texas A\&M University; Ibis, Ibis Biosciences; Pos, positive; Neg, negative; ND, not determined.

${ }^{c}$ PCR-negative samples not shown.

${ }^{d}$ flaB PCR for B. burgdorferi sensu lato (26).

e $16 \mathrm{~S}$ rRNA qPCR for Borrelia spp. (31). $C_{T}$, cycle threshold; RF, relapsing fever group Borrelia.

${ }^{f}$ 16S-23S rRNA intergenic spacer region (IGS) PCR for Borrelia spp. (30).

${ }^{g}$ PCR, electrospray ionization mass spectrometry (IA/PCR/ESI-MS) for Borrelia (32, 33); var., variant.

${ }^{h}$ fla PCR for Borrelia spp. (23).

sent blinded from USAPHC to Ibis Biosciences for analysis using the IA/PCR/ESI-MS assay.

The two pools (P009 and P191) that were negative in all TAMU assays were also negative in the Ibis assay. The four previously Borrelia-negative pools (P184, P187, P220, and P221) and two (131431 and 131570) of the five Borrelia-negative individual samples sent as controls were also negative using the Ibis assay. Three of the individual samples $(131559,131560$, and 131571) determined to be negative at USAPHC produced weak positives for $B$. lonestari in analysis at Ibis Biosciences (Table 2). All five pools (P123, P171, P174, P194, and P211) and four individual samples $(131114,131429,131433$, and 131566) identified as containing $B$. lonestari at TAMU were also positive for this organism at Ibis Biosciences. Pool P189, which was suspect positive for B. burgdorferi in two assays at TAMU was also positive for B. burgdorferi in the Ibis assay using their IA/PCR/ESI-MS assay, but for only three of eight primers: BCT 3515 (rplB gene), BCT 3517 (flagellin gene), and BCT 3519 ( $h b b$ gene). The pool (P084) containing the B. burgdorferi-positive $I$. scapularis tick molecularly identified at TAMU was positive for B. burgdorferi as well as B. lonestari at Ibis Biosciences.

Follow-up testing at USAPHC and TAMU. In order to thoroughly investigate P189, the DNA of the five ticks (131535, $131536,131537,131538$, and 151359) that comprised this pool was analyzed individually both at USAPHC and at TAMU. Two nested PCRs for Borrelia were used at USPAHC: the flaB assay from Clark et al. (26) and the Borrelia genus fla PCR from Barbour et al. (23). In the former assay, all of the individual samples from P189 produced multiple faint bands (laddering) and none had a 437-bp major band; all were scored as negative. Similarly, all sam- 


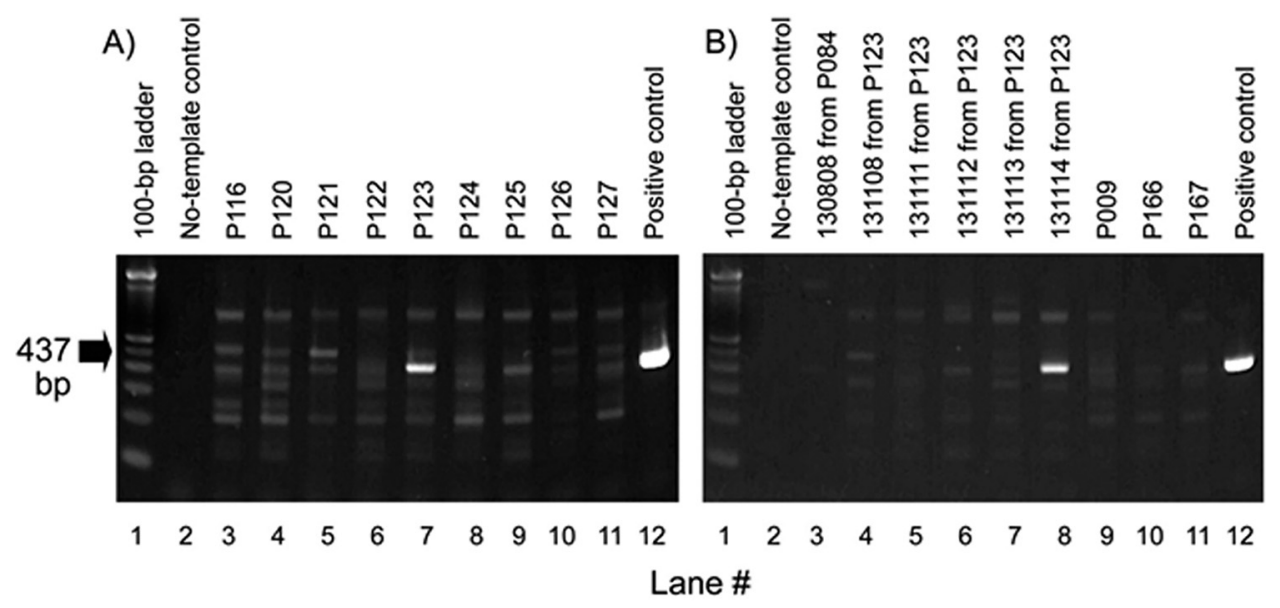

FIG 1 Agarose gels after nested PCR to amplify the Borrelia flaB gene (26) in Amblyomma americanum ticks. Nonspecific binding was present in all tick samples but absent in the negative-control lane (no. 2) and the B. burgdorferi B31 positive-control lane (no. 12). Only samples with bright bands at $437 \mathrm{bp}$ were considered suspect positive in our study. (A) Lanes 3 to 11 correspond to pooled samples of ticks. (B) Lanes 3 to 8 correspond to individual tick samples from previously tested pools, and lanes 9 to 11 are retests of pooled samples. Pooled sample P123 (gel A, lane 7) and individual sample 131114 from P123 (gel B, lane 8 ) were confirmed to be infected with B. lonestari by PCR and sequencing of the IGS PCR product and by IA/PCR/ESI-MS (Table 2).

ples were negative in the generic fla assay. Using the same flaB assay from Clark et al. (26) at the TAMU laboratory, we also obtained a laddering effect in most samples on the gel, indicative of mispriming. One sample (131536) produced a faint band at the diagnostic 437-bp size (in addition to at least one smaller fragment band of the same intensity). Attempts to obtain a DNA sequence from this sample using a direct approach as well as after excising the 437-bp band from the gel were not successful, and the sample was therefore scored as negative.

In silico analysis of the nested-PCR assay. NCBI PrimerBLAST analysis of the nested-PCR assay from Clark et al. (26) indicated that the flagellin genes from multiple Borrelia species could be amplified. For the external reaction, a 475-bp amplicon is expected. The BLAST results yielded amplicons of 445 to $475 \mathrm{bp}$ that corresponded to 19 Borrelia species, including the experimentally identified species $B$. burgdorferi, $B$. andersonii, B. americana, and B. lonestari. For the internal reaction, a 437-bp amplicon is expected. The BLAST results yielded amplicons of 407 to $440 \mathrm{bp}$. All 19 species identified by the external reaction are also potential mispriming loci with the internal reaction.

A more detailed in silico analysis was performed comparing the flaB gene from B. burgdorferi to those of the three experimentally identified cross-reacting Borrelia species. The external and internal primers were also aligned to the sequences. The external primer assay revealed a perfect match to $B$. burgdorferi and $B$. andersonii (due to the degenerate base) and a single mismatch to B. americana. The external reaction has multiple mismatches to the $B$. lonestari sequence, most importantly a mismatch at the $3^{\prime}$-terminal nucleotide in the reverse primer and a mismatch $3 \mathrm{bp}$ from the $3^{\prime}$ terminus of the forward primer. The internal reaction has a perfect match with B. burgdorferi, B. andersonii, and B. americana. This reaction has four mismatches between the internal reverse primer and the $B$. lonestari sequence, including two close to the $3^{\prime}$ end of the primer.

Sensitivity test. The MegAlign comparison of the flagellin sequences found in NCBI GenBank revealed that all four of the Clark primers were an exact match to B. americana SCW-30E. Three of the primers (301F, 737R, and 745R) were $100 \%$ matches to B. andersonii SI-10 and B. bissettii FD-1, although with the $301 \mathrm{~F}$ primer, only $21 / 23$ bases of the primer could be determined. The match of the $280 \mathrm{~F}$ primer to B. andersonii SI- 10 and B. bissettii FD-1 could not be determined as the sequences are unknown; however, the $280 \mathrm{~F}$ primer was an exact match to all of the U.S. sequences of B. bissettii (DN127) and B. andersonii (19857, 21038, and 21123) that were available in NCBI GenBank.

The B. americana SCW-30E flagellin sequence was a 100\% (24/ 24) match with the Barbour FlaLL primer and a 95\% (21/22) match with FlaLS; its matches with FlaRS and FlaRL could not be determined. However, the FlaRS primer was a 100\% match (26/ 26 ) with the one U.S. sequence of $B$. americana (SCW-30 h) that was available in NCBI GenBank. The B. andersonii SI-10 flagellin sequence was a $100 \%$ match $(22 / 22)$ with the Barbour FlaLL primer over the 22/24 bases it could be aligned with, an $86 \%$ (19/ 22) match with FlaLS, a 96\% (25/26) match with FlaRS, and a $92 \%$ (11/12) match with FlaRL over the 11/24 bases it could be aligned with. The B. bissettii FD-1 flagellin sequence was a $100 \%$ match (22/22) with the Barbour FlaLL primer over the 22/24 bases it could be aligned with, a 100\% (22/22) match with FlaLS, and a 92\% (24/26) match with FlaRS, and its match with FlaRL could not be determined. However, the FlaRL primer was a $96 \%$ match (23/24) with the one U.S. sequence of B. bissettii (DN127) that was available in NCBI GenBank.

In the PCR comparison, the flaB primers from Clark (26) and the fla primers from Barbour et al. (23) produced identical results in amplifying increasingly dilute amounts of the three Lyme group spirochetes. The analytical sensitivity (limit of detection [LOD]) of both primer sets in amplifying $B$. americana DNA was determined to be $\sim 0.3 \mathrm{copy} / \mu \mathrm{l}$ (1:10 M dilution). The LOD of both primer sets in detecting $B$. andersonii DNA was $\sim 7$ copies $(1: 1 \mathrm{M}$ dilution), and the LOD of both primer sets in detecting $B$. andersonii DNA was $\sim 6$ copies (1:1 M dilution).

\section{DISCUSSION}

We were unable to confirm any Lyme group Borrelia infection in a detailed investigation of 1,097 A. americanum ticks removed from humans in the southeastern and mid-Atlantic United States. 
Other recent investigations have similarly failed to reveal $B$. burgdorferi in A. americanum. No B. burgdorferi was detected during an extensive molecular characterization of the microbiome of $732 \mathrm{~A}$. americanum adults and nymphs collected in Texas and Missouri (34), and a study of $>3,000$ A. americanum ticks from Georgia using PCR with fla primers from Barbour et al. (23) reported detection only of $B$. lonestari (35). The Barbour primers are designed to amplify all Borrelia spp., and analytical sensitivity testing of these primers in this study has indicated that they can detect the strains of B. americana, B. andersonii, and B. bissettii that have the most mismatches with these primers, suggesting that they can detect all members of each of these species.

Clark et al. (26) have suggested that A. americanum ticks are a significant source of viable B. burgdorferi (sensu lato or sensu stricto). In our hands, however, the flaB nested-PCR assay upon which that suggestion was based produced many faint nonspecific bands, likely indicative of mispriming by amplification of DNA from the tick, human DNA in the tick blood meal, or other organisms in the tick midgut microbiome. Amplification was also inconsistent since samples with major 437-bp bands failed to produce amplicons in repeat testing. Similar inconsistent results (i.e., negative when previously suspect positive or with inconsistent band sizes and numbers) have been reported in another trial of the Clark et al. flaB assay using I. scapularis ticks (36); in that study, as in ours, sequencing attempts of the PCR products of the unknown tick samples failed. This stochastic fluctuation in PCR outcome may be attributable to the low annealing temperature and/or the high primer concentration. The Clark assay utilizes an annealing temperature of $52^{\circ} \mathrm{C}$ for the external reaction and $55^{\circ} \mathrm{C}$ for the internal reaction (26). The optimal annealing temperature in a PCR should be determined empirically, but it must be below the melting temperature $\left(T_{m}\right)$ of the oligonucleotide primers. Results of analysis using the $T_{m}$ calculator indicate that the $T_{m}$ for range for the flaB primers $(26)$ is 60 to $62^{\circ} \mathrm{C}$. However, primer mismatches to the DNA template, such as to the $B$. lonestari flagellin gene, reduce primer $T_{m}$. Including the $B$. lonestari mismatches in the $T_{m}$ calculator resulted in a $T_{m}$ range of 57 to $64^{\circ} \mathrm{C}$. (The $T_{m}$ for some primers increased due to an increase in GC percentage once $\mathrm{A} / \mathrm{T}$ mismatches were removed.) The same analysis could be used for all other potential cross-reacting nontarget species, which may explain the laddering effect of this nested assay. We did not take steps to optimize the PCR primers or protocols described by Clark et al. because our aim was to closely replicate the conditions by which $B$. burgdorferi DNA was reported as being detected in that study. Although these flaB primers were described as being specific for Lyme group Borrelia spp., we used an independent PCR for a different genetic region (30) to amplify and sequence $B$. lonestari from samples that appeared to be suspect positive in the flaB assay (26).

Nucleotide mismatches at the $3^{\prime}$ terminus of a primer significantly reduce polymerase extension. However, a low level of polymerase readthrough can still occur (37), and because PCR results in exponential amplification of a template, even a low level of readthrough can become significant after sufficient PCR cycles. The ability of the Clark primers to amplify $B$. andersonii and $B$. americana has already been experimentally identified $(26,28)$, but the $B$. lonestari result is unexpected. We hypothesize that PCR readthrough from the external reaction explains both the $B$. lonestari positive results, and the lack of repeat positive results. If the readthrough occurs during the first cycles of the external reaction, then sufficient amplicon is produced to have a high rate of amplification in the internal reaction, resulting in a major band of the expected size. The internal reaction should amplify B. lonestari sequences from the external reaction, since the forward internal primer has an exact match to $B$. lonestari sequence, and the reverse primer only shows mismatches at the $5^{\prime}$ end of the primer, which will not affect amplification. However, if readthrough does not occur until a later cycle of the external reaction, insufficient amplicons are produced, resulting in a negative reaction.

Although readthrough and mispriming occur, we would not expect this to be a common event. Therefore, we examined the PCR methods as an additional explanation. The readthrough and mispriming likelihood may have been increased due to the high concentration of primers and low annealing temperature specified by Clark et al. This analysis reveals that the low annealing temperatures of the Clark assay are not very stringent, increasing the probability of mispriming resulting in amplification of B. lonestari and other nonspecific targets. This mispriming has the result of decreasing the sensitivity of these primers so that a higher target copy number is required for amplification to occur. Although Clark et al. performed a BLAST analysis (26), the analysis would not have identified many of the 19 identified potential cross-reacting Borrelia species if they used the reference genome data set or only BLASTed against selected GenBank files. Many Borrelia genomes (including B. lonestari) are not yet fully sequenced. Although we chose the longest deposited $B$. lonestari flagellin sequence for the alignment to B. burgdorferi, we did an additional alignment with $109 \mathrm{~B}$. lonestari flagellin sequences deposited in NCBI Nucleotide (data not shown). Only three records included the outer forward reaction, but five included the inner forward reaction, all of which showed sequence identity. All 109 records included the inner reverse reaction, and 107 records included the outer reverse reaction. Of these alignments, only two did not have sequence identity. This alignment confirmed the accuracy of the sequence we used for the original alignment.

As explained above, the assay from Clark et al. (26) would not be expected to consistently amplify $B$. lonestari; therefore, some $B$. lonestari-positive $A$. americanum samples might have been missed in the initial screen at USAPHC using only the Clark primers. Testing at TAMU and Ibis Biosciences using assays designed to amplify $B$. lonestari detected this organism in samples that had produced negative or inconsistent results using the Clark primers at USAPHC (Table 2). However, the low prevalence of infection with $B$. lonestari detected in these samples is congruent with previous surveillance $(2,8,21,24,34,35)$. For example, in our program from 1997 to 2010, a total of 18,546 A. americanum ticks were tested with nested and real-time primers that were capable of amplifying B. lonestari, and 195/17,226 (1.1\%) of adults and nymphs and $4 / 1,320$ larvae $(0.3 \%$ minimum infection prevalence) were positive $(2,8,20)$.

Ibis Biosciences reported low-level detection of B. burgdorferi DNA in a pooled sample of $A$. americanum DNA (sample P189) by three of the eight primer pairs capable of detecting B. burgdorferi. This pool had also been weakly positive for B. burgdorferi in the two assays at TAMU. The PCR signal was very different from that provided by B. burgdorferi-infected I. scapularis ticks; furthermore, B. burgdorferi sequences were not detected in any of the individual samples in the pool. We suggest the weak result in our assays may reflect low-level contamination of P189, possibly introduced at the DNA isolation and pool-forming step, or the am- 
plification of the remnants of an infected blood meal from a previous life stage. All five samples in this pool were single ticks from Ft. Pickett, VA; two were nymphs, two were females, one was a male, and all were unengorged and removed from humans. A few B. burgdorferi-infected I. scapularis ticks have been submitted to the HTTKP from Ft. Pickett, demonstrating that the pathogen is indeed circulating in that environment (E. Stromdahl, unpublished data). Other Lyme group Borrelia species, B. americana, B. andersonii, B. bissetti, etc., were not detected.

To summarize, we were unable to confirm any Lyme group Borrelia infection in A. americanum ticks removed from humans in the southeastern and mid-Atlantic United States. In our investigation, we utilized the flaB primers and thermal cycling parameters of Clark et al. because this diagnostic tool was associated with the reported finding of Lyme group Borrelia DNA in a small number of $A$. americanum ticks and blood samples from humans reported to have been fed upon by A. americanum (26). In our investigation, this flaB assay produced indistinct and inconsistent results in A. americanum ticks, and the samples that produced major bands at the expected fragment size were confirmed to be positive for B. lonestari DNA (but not Lyme group Borrelia DNA) in multiple different assays. In agreement with decades of previous research, we therefore conclude that human-biting $A$. americanum ticks are not a vector of $B$. burgdorferi. The conclusion of Clark et al. that A. americanum is infected with Lyme group Borrelia spp. is based on the detection of flaB gene sequences alone. Given that this finding is in striking contrast to decades of previous investigations of the topic, a more rigorous approach would have been to more fully characterize the organism through the amplification and sequencing of multiple genes or verification by culturing of the organism, to provide more convincing evidence of identification (38). Conclusions based on inadequate evidence exacerbate public confusion: findings of Borrelia spp. in novel tick species should in future always be supported by characterization of multiple gene targets.

\section{ACKNOWLEDGMENTS}

We thank Christopher Johnson for technical support and Zia Mehr for technical review.

The views expressed in this article are those of the authors and do not reflect the official policy or position of the Department of the Army, Department of Defense or the U.S. Government. Some of the authors, as employees of the U.S. Government (E.Y.S., R.M.N., C.E.E., M.P.M., M.A.V., and J.A.G.), conducted the work as part of their official duties. M.W.E., C.D.C., and H.E.C. are employees of Ibis Biosciences, an Abbott company, which developed the PCR/ESI-MS and IA/PCR/ESI-MS assays and instrumentation used in these studies.

\section{REFERENCES}

1. Rudenko N, Golovchenko M, Grubhoffer L, Oliver JH, Jr. 2011. Updates on Borrelia burgdorferi sensu lato complex with respect to public health. Ticks Tick Borne Dis 2:123-128. http://dx.doi.org/10.1016/j.ttbdis .2011.04.002.

2. Stromdahl EY, Williamson PC, Kollars TM, Jr, Evans SR, Barry RK, Vince MA, Dobbs NA. 2003. Evidence of Borrelia lonestari DNA in Amblyomma americanum (Acari: Ixodidae) removed from humans. J Clin Microbiol 41:5557-5562. http://dx.doi.org/10.1128/JCM.41.12.5557-5562.2003.

3. Dworkin MS, Schwan TG, Anderson DE, Jr, Borchardt SM. 2008. Tick-borne relapsing fever. Infect Dis Clin North Am 22:449-468. http: //dx.doi.org/10.1016/j.idc.2008.03.006.

4. Chowdri HR, Gugliotta JL, Berardi VP, Goethert HK, Molloy PJ, Sterling SL, Telford SR. 2013. Borrelia miyamotoi infection presenting as human granulocytic anaplasmosis: a case report. Ann Intern Med 159:2127. http://dx.doi.org/10.7326/0003-4819-159-1-201307020-00005.
5. James AM, Liveris D, Wormser GP, Schwartz I, Montecalvo MA, Johnson BJ. 2001. Borrelia lonestari infection after a bite by an Amblyomma americanum tick. J Infect Dis 183:1810-1814. http://dx.doi.org/10 $.1086 / 320721$

6. Wormser GP, Masters E, Liveris D, Nowakowski J, Nadelman RB, Holmgren D, Bittker S, Cooper D, Wang G, Schwartz I. 2005. Microbiologic evaluation of patients from Missouri with erythema migrans. Clin Infect Dis 40:423-428. http://dx.doi.org/10.1086/427289.

7. Wormser GP, Masters E, Nowakowski J, McKenna D, Holmgren D, Ma K, Ihde L, Cavaliere LF, Nadelman RB. 2005. Prospective clinical evaluation of patients from Missouri and New York with erythema migranslike skin lesions. Clin Infect Dis 41:958-965. http://dx.doi.org/10.1086 1432935.

8. Stromdahl EY, Hickling GJ. 2012. Beyond Lyme: aetiology of tick-borne human diseases with emphasis on the south-eastern United States. Zoonoses Public Health 59(Suppl 2):S48-S64. http://dx.doi.org/10.1111/j .1863-2378.2012.01475.x.

9. Springer YP, Eisen L, Beati L, James AM, Eisen RJ. 2014. Spatial distribution of counties in the continental United States with records of occurrence of Amblyomma americanum (Ixodida: Ixodidae). J Med Entomol 51:342-351. http://dx.doi.org/10.1603/ME13115.

10. Mather TN, Telford SR, III, Moore SI, Spielman A. 1990. Borrelia burgdorferi and Babesia microti: efficiency of transmission from reservoirs to vector ticks (Ixodes dammini). Exp Parasitol 70:55-61. http://dx.doi .org/10.1016/0014-4894(90)90085-Q.

11. Mukolwe SW, Kocan AA, Barker RW, Kocan KM, Murphy GL. 1992. Attempted transmission of Borrelia burgdorferi (Spirochaetales: Spirochaetaceae) (JDI strain) by Ixodes scapularis (Acari: Ixodidae), Dermacentor variabilis, and Amblyomma americanum. J Med Entomol 29:673-677. http://dx.doi.org/10.1093/jmedent/29.4.673.

12. Oliver JH, Jr, Chandler FW, Jr, Luttrell MP, James AM, Stallknecht DE, McGuire BS, Hutcheson HJ, Cummins GA, Lane RS. 1993. Isolation and transmission of the Lyme disease spirochete from the southeastern United States. Proc Natl Acad Sci U S A 90:7371-7375. http://dx.doi.org/10.1073 /pnas.90.15.7371.

13. Piesman J, Happ CM. 1997. Ability of the Lyme disease spirochete Borrelia burgdorferi to infect rodents and three species of human-biting ticks (blacklegged tick, American dog tick, lone star tick) (Acari:Ixodidae). J Med Entomol 34:451-456. http://dx.doi.org/10.1093/jmedent/34.4.451.

14. Piesman J, Sinsky RJ. 1988. Ability to Ixodes scapularis, Dermacentor variabilis, and Amblyomma americanum (Acari: Ixodidae) to acquire, maintain, and transmit Lyme disease spirochetes (Borrelia burgdorferi). J Med Entomol 25:336-339. http://dx.doi.org/10.1093/jmedent/25.5.336.

15. Ryder JW, Pinger RR, Glancy T. 1992. Inability of Ixodes cookei and Amblyomma americanum nymphs (Acari: Ixodidae) to transmit Borrelia burgdorferi. J Med Entomol 29:525-530. http://dx.doi.org/10.1093 /jmedent/29.3.525.

16. Sanders FH, Oliver JH. 1995. Evaluation of Ixodes scapularis, Amblyomma americanum, and Dermacentor variabilis (Acari, Ixodidae) from Georgia as vectors of a Florida strain of the Lyme-disease spirochete, Borrelia burgdorferi. J Med Entomol 32:402-406. http://dx.doi.org/10.1093 /jmedent/32.4.402.

17. Ledin KE, Zeidner NS, Ribeiro JM, Biggerstaff BJ, Dolan MC, Dietrich G, Vredevoe L, Piesman J. 2005. Borreliacidal activity of saliva of the tick Amblyomma americanum. Med Vet Entomol 19:90-95. http://dx.doi.org /10.1111/j.0269-283X.2005.00546.x.

18. Zeidner N, Ullmann A, Sackal C, Dolan M, Dietrich G, Piesman J, Champagne D. 2009. A borreliacidal factor in Amblyomma americanum saliva is associated with phospholipase A(2) activity. Exp Parasitol 121: 370-375. http://dx.doi.org/10.1016/j.exppara.2009.01.002.

19. Stromdahl EY, Evans SR, O’Brien JJ, Gutierrez AG. 2001. Prevalence of infection in ticks submitted to the human tick test kit program of the US Army Center for Health Promotion and Preventive Medicine. J Med Entomol 38:67-74. http://dx.doi.org/10.1603/0022-2585-38.1.67.

20. Stromdahl EY, Vince MA, Billingsley PM, Dobbs NA, Williamson PC. 2008. Rickettsia amblyommii infecting Amblyomma americanum larvae. Vector Borne Zoonotic Dis 8:15-24. http://dx.doi.org/10.1089/vbz.2007 .0138 .

21. Bacon RM, Pilgard MA, Johnson BJ, Piesman J, Biggerstaff BJ, Quintana M. 2005. Rapid detection methods and prevalence estimation for Borrelia lonestari glpQ in Amblyomma americanum (Acari: Ixodidae) pools of unequal size. Vector Borne Zoonotic Dis 5:146-156. http://dx.doi .org/10.1089/vbz.2005.5.146. 
22. Bacon RM, Pilgard MA, Johnson BJB, Raffel SJ, Schwan TG. 2004. Glycerophosphodiester phosphodiesterase gene $(g l p Q)$ of Borrelia lonestari identified as a target for differentiating Borrelia species associated with hard ticks (Acari: Ixodidae). J Clin Microbiol 42:2326-2328. http://dx.doi .org/10.1128/JCM.42.5.2326-2328.2004.

23. Barbour AG, Maupin GO, Teltow GJ, Carter CJ, Piesman J. 1996. Identification of an uncultivable Borrelia species in the hard tick Amblyomma americanum: possible agent of a Lyme disease-like illness. J Infect Dis 173:403-409. http://dx.doi.org/10.1093/infdis/173.2.403.

24. Williamson PC, Billingsley PM, Teltow GJ, Seals JP, Turnbough MA, Atkinson SF. 2010. Borrelia, Ehrlichia, and Rickettsia spp. in ticks removed from persons, Texas, USA. Emerg Infect Dis 16:441-446. http://dx.doi .org/10.3201/eid1603.091333.

25. Masters EJ. 2006. Lyme-like illness currently deserves Lyme-like treatment. Clin Infect Dis 42:580-582. http://dx.doi.org/10.1086/500018.

26. Clark KL, Leydet B, Hartman S. 2013. Lyme borreliosis in human patients in Florida and Georgia, USA. Int J Med Sci 10:915-931. http://dx doi.org/10.7150/ijms.6273.

27. Stromdahl EY, Jiang J, Vince M, Richards AL. 2011. Infrequency of Rickettsia rickettsii in Dermacentor variabilis removed from humans, with comments on the role of other human-biting ticks associated with spotted fever group Rickettsiae in the United States. Vector Borne Zoonotic Dis 11:969-977. http://dx.doi.org/10.1089/vbz.2010.0099.

28. Clark KL, Leydet BF, Threlkeld C. 2014. Geographical and genospecies distribution of Borrelia burgdorferi sensu lato DNA detected in humans in the USA. J Med Microbiol 63:674-684. http://dx.doi.org/10.1099/jmm.0 .073122-0.

29. Beati L, Keirans JE. 2001. Analysis of the systematic relationships among ticks of the genera Rhipicephalus and Boophilus (Acari: Ixodidae) based on mitochondrial $12 \mathrm{~S}$ ribosomal DNA gene sequences and morphological characters. J Parasitol 87:32-48. http://dx.doi.org/10.1645/0022 -3395(2001)087[0032:AOTSRA]2.0.CO;2.

30. Bunikis J, Garpmo U, Tsao J, Berglund J, Fish D, Barbour AG. 2004 Sequence typing reveals extensive strain diversity of the Lyme borreliosis agents Borrelia burgdorferi in North America and Borrelia afzelii in Europe. Microbiology 150:1741-1755. http://dx.doi.org/10.1099/mic.0.26944-0.

31. Tsao JI, Wootton JT, Bunikis J, Luna MG, Fish D, Barbour AG. 2004.
An ecological approach to preventing human infection: vaccinating wild mouse reservoirs intervenes in the Lyme disease cycle. Proc Natl Acad Sci, U S A 101:18159-18164. http://dx.doi.org/10.1073/pnas.0405763102.

32. Eshoo MW, Crowder CC, Rebman AW, Rounds MA, Matthews HE, Picuri JM, Soloski MJ, Ecker DJ, Schutzer SE, Aucott JN. 2012. Direct molecular detection and genotyping of Borrelia burgdorferi from whole blood of patients with early Lyme disease. PLoS One 7:e36825. http://dx .doi.org/10.1371/journal.pone.0036825.

33. Crowder CD, Matthews H, Schutzer S, Rounds MA, Luft BJ, Nolte O, Campbell SR, Phillipson CA, Li F, Sampath R, Ecker DJ, Eshoo MW. 2010. Genotypic variation and mixtures of Lyme Borrelia in Ixodes ticks from North America and Europe. PLoS One 5:E10650-E10659. http://dx .doi.org/10.1371/journal.pone.0010650.

34. Yuan DT. 2010. A metagenomic study of the tick midgut. UT GSBS dissertations and theses. Paper 85. University of Texas Graduate School of Biomedical Sciences, Houston, TX. http://digitalcommons.library.tmc .edu/utgsbs_dissertations/85.

35. Gleim ER. 2013. The effects of long-term prescribed burning on ticks and tick-borne pathogen prevalence. Ph.D. dissertation. The University of Georgia, Athens, GA.

36. Mays SE, Hendricks BM, Paulsen DJ, Houston AE, Trout Fryxell RT. 2014. Prevalence of five tick-borne bacterial genera in adult Ixodes scapularis removed from white-tailed deer in western Tennessee. Parasit Vectors 7:473. http://dx.doi.org/10.1186/s13071-014-0473-y.

37. Nikolausz M, Chatzinotas A, Táncsics A, Imfeld G, Kästner M. 2009. Evaluation of single-nucleotide primer extension for detection and typing of phylogenetic markers used for investigation of microbial communities. Appl Environ Microbiol 75:2850-2860. http://dx.doi.org/10.1128/AEM $.01910-08$

38. Margos G, Gatewood AG, Aanensen DM, Hanincová K, Terekhova D, Vollmer SA, Kurtenbach K, Cornet M, Piesman J, Donaghy Bormane A, Hurn M, Feil E, Fish D, Casjens S, Wormser G, Schwartz I, Kurtenbach K. 2008. MLST of housekeeping genes captures geographic population structure and suggests a European origin of Borrelia burgdorferi. Proc Natl Acad Sci U S A 105:8730-8735. http://dx.doi.org/10.1073/pnas .0800323105 